\title{
GENERAL THEOREMS ON EXPONENTIAL AND ROSENTHAL'S INEQUALITIES AND ON COMPLETE CONVERGENCE
}

\section{István FAZEKas, SÁndor Pecsora And Bettina PorvázSnYik}

Abstract. Exponential inequalities are obtained under general conditions. Then it is shown that an exponential inequality implies both Rosenthal's inequality and complete convergence of sums of random variables. The general results are applied to weakly orthant dependent sequences.

Mathematics subject classification (2010): 60F15, 60G50.

Keywords and phrases: Exponential inequality, Rosenthal's inequality, strong law of large numbers, complete convergence, acceptable random variables, weakly orthant dependent sequences.

\section{REFERENCES}

[1] R. G. Antonini, Y. Kozachenko, A. Volodin, Convergence of series of dependent $\varphi$-subGaussian random variables, J. Math. Anal. Appl. 338, no. 2 (2008), 1188-1203.

[2] L. E. BAuM, M. KATZ, Convergence rates in the law of large numbers, Trans. Amer. Math. Soc. 120 (1965), 108-123.

[3] N. H. Bingham, C. M. Goldie, J. L. Teugels, Regular variation, Cambridge University Press, Cambridge, 1987.

[4] T. C. Christofides, M. Hadjikyriakou, Exponential inequalities for $N$-demimartingales and negatively associated random variables, Statist. Probab. Lett. 79, no. 19 (2009), 2060-2065.

[5] P. ERdôs, On a theorem of Hsu and Robbins, Ann. Math. Statistics 20 (1949), 286-291.

[6] I. FAZEKAS, Convergence rates in the law of large numbers for arrays, Publ. Math. Debrecen 41, no. 1-2 (1992), 53-71.

[7] I. FAZEKAS, On a general approach to the strong laws of large numbers, J. Math. Sci. (N. Y.) 200, no. 4 (2014), 411-423.

[8] I. FAZEKAS, O. I. KLESOV, A general approach to the strong laws of large numbers, Teor. Veroyatnost. i Primenen. 45 (2000), no. 3, 568-583, Theory Probab. Appl. 45 (2002), no. 3, 436-449.

[9] I. Fazekas, A. G. Kukush, T. TómÁcs, On the Rosenthal inequality for mixing fields, Ukrainian Math. J. 52, no. 2 (2000), 305-318.

[10] W. FeLLER, An introduction to probability theory and its applications, vol. II, Wiley, New York, 1971.

[11] D. H. Fuk, S. V. NaGAev, Probabilistic inequalities for sums of independent random variables, Teor. Verojatnost. i Primenen. 16 (1971), 660-675.

[12] ShIXIN Gan, PIng Yan Chen, Dehua QIU, Rosenthal inequality for NOD sequences and its applications, Wuhan Univ. J. Nat. Sci. 16, no. 3 (2011), 185-189.

[13] A. Gut, Complete convergence for arrays, Period. Math. Hungar. 25, no. 1 (1992), 51-75.

[14] A. Gut, Probability: a graduate course, Springer, New York, 2013.

[15] W. Hoeffoing, Probability inequalities for sums of bounded random variables, J. Amer. Statist. Assoc. 58 (1963), 13-30.

[16] P. L. Hsu, H. Robbins, Complete convergence and the law of large numbers, Proc. Nat. Acad. Sci. U. S. A. 33 (1947), 25-31.

[17] T. C. HU, F. MóRICZ, R. L. TAYLOR, Strong laws of large numbers for arrays of rowwise independent random variables, Acta Math. Hungar. 54, no. 1-2 (1989), 153-162.

[18] F. MóRICZ, Exponential estimates for the maximum of partial sums, I, Sequences of rv's, Acta Math. Acad. Sci. Hungar. 33, no. 1-2 (1979), 159-167. 
[19] S. V. Nagaev, I. F. Pinelis, Some inequalities for the distribution of sums of independent random variables, Teor. Veroyatn. Primen. 22 (1977), 254-263.

[20] V. V. Petrov, Limit theorems of probability theory. Sequences of independent random variables, The Clarendon Press, Oxford University Press, New York, 1995.

[21] QI Man Shao, Complete convergence for $\alpha$-mixing sequences, Statist. Probab. Lett. 16, no. 4 (1993), 279-287.

[22] Aiting Shen, Shuhe Hu, Andrei Volodin, Xuejun Wang, Some exponential inequalities for acceptable random variables and complete convergence, J. Inequal. Appl. 2011:142 (2011), 10 pp.

[23] W. F. Stout, Almost sure convergence, Probab. Math. Statistics, vol. 24, Academic Press, New YorkLondon, 1974.

[24] Soo HaK Sung, Moment inequalities and complete moment convergence, J. Inequal. Appl., Art. ID 271265 (2009), 14 pp.

[25] KaiYong WAng, Yuebao Wang, QIngWU Gao, Uniform asymptotics for the finite-time ruin probability of a dependent risk model with a constant interest rate, Methodol. Comput. Appl. Probab. 15, no. 1 (2013), 109-124.

[26] Xinghui Wang, Shuhe Hu, Complete convergence and complete moment convergence for a class of random variables, J. Inequal. Appl. 2012: 229 (2012), 12 pp.

[27] Xuejun Wang, Chen Xu, Tien-Chung Hu, Andrei Volodin, Shuhe Hu, On complete convergence for widely orthant-dependent random variables and its applications in nonparametric regression models, TEST 23, no. 3 (2014), 607-629.

[28] Yuebao WANG, YaWei Li, QINGWU GaO, On the exponential inequality for acceptable random variables, J. Inequal. Appl. 2011: 40 (2011), 10 pp.

[29] Yongfeng Wu, Mei Guan, Convergence properties of the partial sums for sequences of end random variables, J. Korean Math. Soc. 49, no. 6 (2012), 1097-1110.

[30] Yongfeng Wu, Mingzhu Song, Chunhua Wang, Complete Moment Convergence and Mean Convergence for Arrays of Rowwise Extended Negatively Dependent Random Variables, Scientific World Journal, Article ID 2014:478612 (2014), doi: 10.1155/2014/478612. 